\title{
Reliability of Youtube Videos On Knee Joint Injection
}

\author{
Sezai Özkan ${ }^{1 *}$, Cihan Adanaş $^{1}$, Şeyhmus Kaplan ${ }^{2}$, Volkan Şah $^{2}$ \\ ${ }^{1}$ Department of Orthopedics and Traumatology, Van YuzuncuYil University Hospital, Van, Turkey \\ ${ }^{2}$ Department of Sports Medicine, Van YuzuncuYil University Hospital, Van, Turkey
}

\begin{abstract}
Intra-articular injection requires a visual learning and the knee is the most commonly used joint for this application. YouTube videos have become a source of visual learning. However, there is a obscurity whether YouTube videos on medical education provide safe and useful information. Therefore, this study aim to evaluate the reliability of YouTube videos on knee joint injection according to the current approach.

The term "knee joint injection technique" was used to search the related YouTube videos. The videos were grouped in terms of the uploader, uploading years, and the number of views. The procedure was evaluated and scored for positioning of the patient, palpation of the landmarks, washing hands and wearing gloves, needle approach and entry, drug injection, removing the needle and placing the sponge and finally bandaging.

Of the 61 videos, only $11(18 \%)$ had enough quality. The score of videos uploaded by an institution was significantly higher than others $(\mathrm{p}=0.006)$. The score of videos viewed over $(n=31)$ and lower $(n=30)$ than 10000 times was 4.5 (IQR $2.0)$ and 3.5 (IQR 2.0), respectively $(\mathrm{p}=0.018)$. There was no statistical significance between the groups according to uploading years $(\mathrm{p}=0.694)$.

YouTube videos are not enough qualified enaugh reliable visual learning of knee joint injection. The institutional YouTube videos are more educational sources than others. YouTube videos with higher views count also have higher reli ability.
\end{abstract}

Keywords: Knee, injection, reliability, YouTube, education

\section{Introduction}

There is a debate about the advantages and disadvantages of digital medical education. Some authors advocate the advantages of digital medical education $(1,2)$; on the other hand, others warn us about losing professionalism (3). Since YouTube was established in early 2005, it has become the most popular and successful internet-based videosharing website (4). All around the world, 1.300.000.000 people are using YouTube every minute, 300 hours of videos are uploaded on YouTube, and finally, about five billion videos are watched on YouTube every day (4). Medical videos are not outside of those, and therefore YouTube has also become a large source of information on medical education.

The knee, the largest hinge joint in the human body, is the most common and the most comfortable body region for the physician to apply intra-articular injection (5). The proper technique requires not only theoretical knowledge but also requires visual learning. Besides this, educational videos have some benefits, such as using time efficiently and reducing technical errors $(6,7)$. So the videos that teach invasive procedures are commonly used to avoid possible mistakes. However, there is great obscurity whether these videos provide safe and useful information for health care professionals.

This study aims to evaluate the reliability of YouTube videos on knee joint injections according to the current approach in the literature $(8,9)$.

\section{Material and Method}

The search was done by using the term "knee joint injection technique". The 383 videos listed on the main page were watched and evaluated. Our exclusion criteria consisted of irrelevant video content, all languages except English, commercial or advertising videos, funny videos, recurrent content, interviews, news, course or seminar videos, and the videos that were uploaded before 2010 (Figure 1). We divide the videos into three groups according to uploading years. The first group was from 2010 to 2012, the second was from 2013 to 2015, and the last group was from 2016 to March 30, 2018.

\footnotetext{
*Corresponding Author: Sezai Özkan, MD, Department of Orthopedics and Traumatology, Van Yuzuncu Yil University Hospital, Van, Turkey

E-mail: doktorsezai@hotmail.com, Phone: 90 (505) 4877170
}

ORCID ID: Sezai Özkan: 0000-0003-4444-6939, Cihan Adanaş: 0000-0002-7490-5270, Şeyhmus Kaplan: 0000-0002-3652-6077, Volkan Şah: 0000-0002-2853-2266

Received: 09.07.2020, Accepted: 21.04.2021 


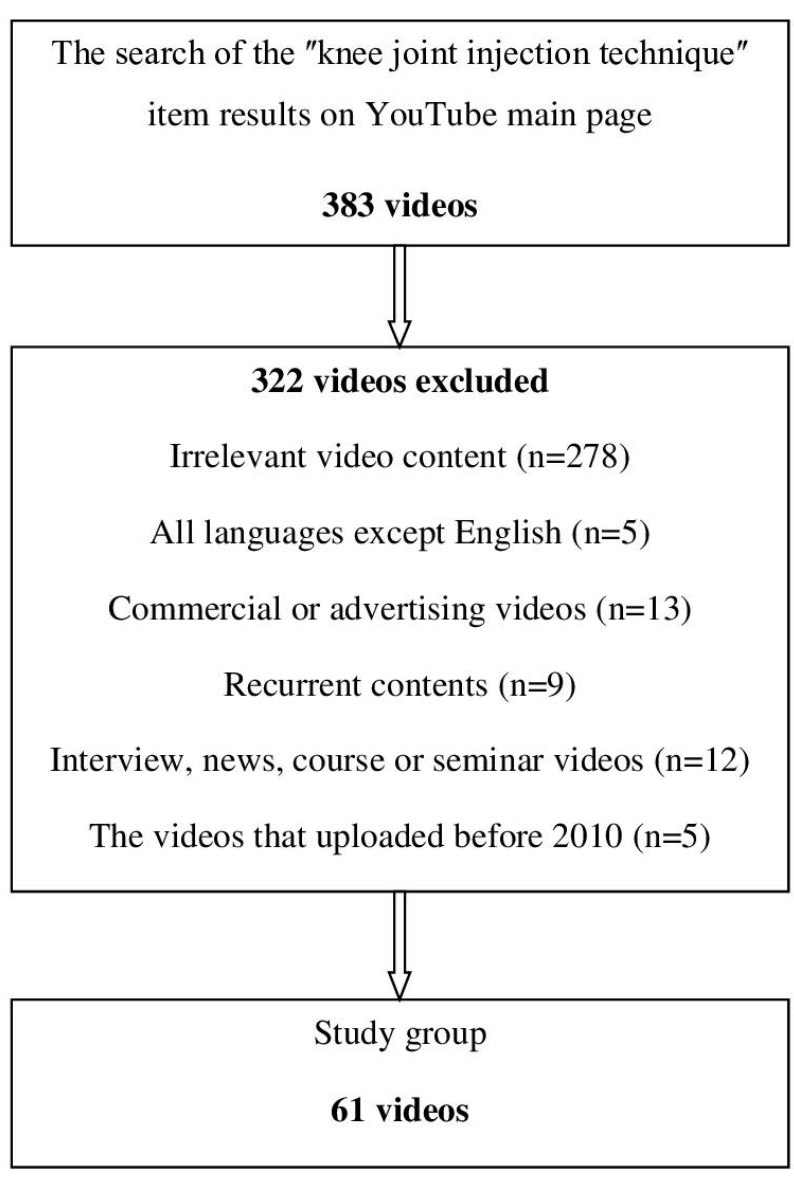

Fig. 1. Flowchart of the search

All videos matched our criteria examined in terms of the uploader, uploading years, number of views, duration, demonstration model of the procedure (human or mannequin model), and local anesthetic proposal. Additionally, the process was evaluated and scored for positioning the patient, palpation of the landmarks, hand washing, wearing gloves, needle approach and entry, drug injection, removing the needle and placing the sponge, and finally bandaging (Table 1). These evaluations were made by two physicians, an orthopedic surgeon and a sports medicine physician, separately. Each physician rated the videos from 0 to 7 points independently of each other. The rating was based on the literature knowledge that specifically reviewed this topic $(8,9)$. We categorized the scores like 6-7 points are enough qualified; 4-5 points are mediocre videos, and finally, lower than 4 points are pretty inadequate (10) (Table 1).

Statistical Analysis: Statistical analysis was made using SPSS 20.0 (IBM SPSS Statistics, Version 20.0. Armonk, NY: IBM Corp). Numeric variable data are represented as median (IQR-interquartile ratio) and frequent variable data as rates. For twogroup comparisons, the Mann-Whitney $\mathrm{U}$ test was

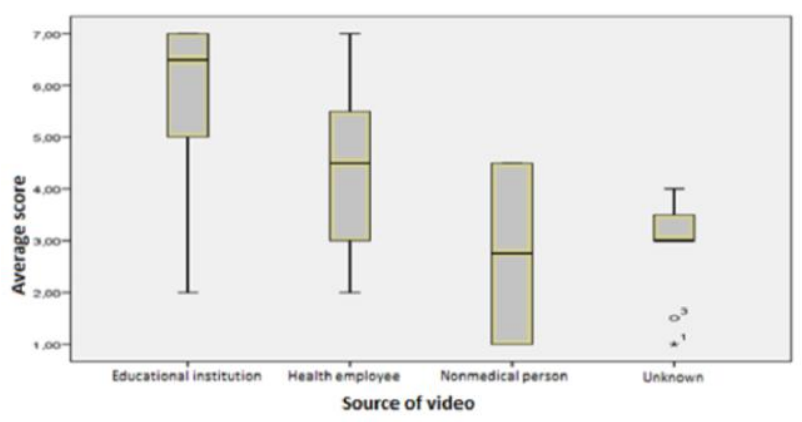

Fig. 2. The Average Scores According To The Uploaders

used, and higher than two-group comparisons were made by using Kruskal-Wallis test. ShapiroWilk test was used for normality tests. The $\mathrm{p}$ values that smaller than 0.05 were accepted as statistical significance.

\section{Results}

After searching with the term "knee joint injection technique", there were 383 videos on ' 'YouTube's main page. Three hundred twenty-two of them were excluded because they matched with exclusion criteria, so only 61 videos of them were included in this study. Of the 61 videos, 44 $(72.1 \%)$ had been uploaded by health professionals, six $(9.8 \%)$ had been uploaded by an institution (university, society, association, clinic, etc.), nine $(14.8 \%)$ videos' uploaders were unknown, and two $(3.3 \%)$ uploader were users other than health professionals. The majority of uploaders $(96.7 \%, \mathrm{n}=59)$ had preferred humans as a descriptive model of the procedure. Only two uploaders $(3.3 \%)$ had used, mannequin model for the invasive procedure stages. Only one video $(1.6 \%)$ consisted of incorrect information. This video had been uploaded by non-medical personnel. He was showing knee joint injection technique irrelevantly with the guidelines. He was showing injection on himself at home and in nonsterile conditions. Videos' distributions according to their content adequacy were as follows: the number of qualified, mediocre, and entirely inadequate videos were 11 (18\%), 25 (41\%), and 25 (41\%), respectively. Local anesthetic was recommended in 17 videos $(27.9 \%)$.

The median scores were 6.5 (IQR 2.75) for the institutional uploader, 4.5 (IQR 2.5) for the health professional uploader, 2.75 for non-medical personnel, and 3.0 (IQR 1.5) for the unknown uploader. The videos uploaded by an institution had significantly higher median scores compared to those uploaded by others $(\mathrm{p}=0.006$ ). (Figure 2 )

The number of videos viewed over 10000 was 31 
Table 1. The Scoring Parameters Chart

\begin{tabular}{lc}
\hline Score parameters & Score \\
\hline Positioning of the patient & 1 \\
Palpation of the landmarks & 1 \\
Washing hands and wearing gloves & 1 \\
Needle approach and entry & 1 \\
Drug injection & 1 \\
Removing the needle and placing the sponge & 1 \\
Bandaging & 1 \\
Score classification & Total score \\
Enough qualified & $6-7$ \\
Mediocre & $4-5.5$ \\
Quite inadequate & $<4$ \\
\hline
\end{tabular}

Table 2. The Comparison of Video Scores According To Variables

\begin{tabular}{lcccc}
\hline Uploader Type & $\mathrm{n}$ & $\%$ & Median score (IQR) & $\mathrm{p}$-value \\
\hline $\begin{array}{l}\text { Institution (university, society, } \\
\text { association, clinic etc.) }\end{array}$ & 6 & 9.8 & $6.5(2.75)$ & 0.006 \\
$\begin{array}{l}\text { Healthcare personnel (doctors, nurses, } \\
\text { etc.) }\end{array}$ & 44 & 72.1 & $4.5(2.5)$ & \\
Individuals other than healthcare & 2 & 3.3 & 2.75 & \\
personnel & 9 & 14.8 & $3(1.5)$ & \\
Unknown (not classified) & $\mathrm{n}$ & $\%$ & Median score (IQR) & $\mathrm{p}$-value \\
View Count & 31 & 50.8 & $3.5(2.0)$ & 0.018 \\
$\geq 10,000$ & 30 & 49.2 & $4.5(2.0)$ & \\
$<10,000$ & $\mathrm{n}$ & $\%$ & Median score (IQR) & $\mathrm{p}$-value \\
Uploading Years & 18 & 26.2 & 4.5 & 0.694 \\
$2010-12$ & 28 & 45.9 & 3.75 & \\
$2013-15$ & 17 & 27.9 & 4.5 & \\
$2016-18$ & & & & \\
\hline
\end{tabular}

$(50.8 \%)$, and $30(49.2 \%)$ videos had been viewed by fewer than 10000 users. The median score of the videos that were viewed over $(n=31)$ and lower $(\mathrm{n}=30)$ than 10000 times was 4.5 (IQR 2.0) and 3.5 (IQR 2.0), respectively ( $\mathrm{p}=0.018$ ).

The median scores of them were 4.5 (IQR 2.25), 3.75 (IQR 2.5), and 4.5 (IQR 1.75), respectively. There was no statistical significance between the groups $(\mathrm{p}=0.694)$.

\section{Discussion}

Because many invasive medical procedures require visual learning, health students and health professionals often benefit from YouTube videos. One of the frequently applied invasive medical procedures is knee joint injection. They are also considering the importance of visual learning in medical education the limited to digital media professionalism. Therefore, with the high popularity of YouTube, we wanted to evaluate the scientific reliability of YouTube videos on knee joint injection techniques.

In our study, although health workers uploaded the vast majority of videos $(72.1 \%)$, only $18 \%$ of them were qualified enough. The good thing was that only one video consisted of incorrect information. Additionally, we have also found a meaningful relationship between the quality of video content and its number of views. But the most important factor affecting the quality was the uploader. The score was higher when the uploader was an institution (university, society, association, clinic, etc.). Finally, we could not find qualify difference between new and old videos. 
In many articles investigating the reliability of YouTube videos, authors had found that when the uploader was an institution, the quality of video content becomes better and serves more accurate information (7,11-14). Our study also supports this data. However, although the number of videos including incorrect information was very small $(1.6 \%)$, there is always a significant potential risk of spreading inaccurate information due to the lack of a refereeing mechanism. Additionally, this risk may be greater if there was no linear relationship between the view count and accurate information score.

When the video contents were evaluated, only $18 \%$ of them were was enough qualified. This ratio is unacceptable for the general quality of any topic. For example, in a study investigating lumbar puncture and neuraxial block techniques, the authors found less than $50 \%$ of videos gave essential information on the procedure and sterility (15). However, these rates demonstrate differences between studies that deal with different subjects $(7,16-19)$. Thus, some authors advise using more technical terms when searching on YouTube to reach more qualified videos (19).

There are limited studies related to the current topic (19-21). Fischer et al.(19) have found YouTube videos' poor overall educational quality on knee arthrocentesis. Karim et al.(20) have concluded that YouTube videos on knee arthrocentesis cannot replace traditional instructor-led learning periods in medical students' education. Finally, Kucukakkas et al.(21) had reported an insufficiency and discrepancy about YouTube content for Web-based learning intraarticular injection even though when healthcare professionals published it.

YouTube accesses are free, easy, and do not need a registration procedure. Also, the videos could be watched anytime and anywhere repeatedly. As a result, it is especially attractive for students. However, the classical method is still important in education (22). Web-based education has already begun to compete with classical training on some issues (23). On the other side, accessing correct information is also indispensable, especially in health. Therefore, this topic should be discussed and be bound to an acceptable and feasible outcome. Perhaps "YouTube Academic" including referee process is necessary.

A major limitation of the current study is the YouTube nature that gives instant information that changes according to the search date and time. In addition, it is possible that we could not reach unlimited data due to search terms and exclusion criteria.

In conclusion, e-learning videos are important in health. However, there is a justifiable concern regarding reliability. Specifically, YouTube videos on knee joint injection techniques are not qualified enough. Videos' quality and the number of views are linearly proportional. The quality of videos uploaded by educational institutions is higher in terms of correct information and reliability.

\section{References}

1. Lockyer J, Sargeant J, Curran V, Fleet L. The transition from face-to-face to online CME facilitation. Med Teach 2006; 28: 625-630.

2. Ozuah PO. Undergraduate medical education: thoughts on future challenges. BMC Med Educ 2002; 2: 8.

3. Farnan JM, Paro JA, Higa J, Edelson J, Arora VM. The YouTube generation: implications for medical professionalism. Perspect Biol Med 2008; 51: 517-524.

4. "37 Mind Blowing YouTube Facts, Figures and Statistics-2018" Available from: https:// fortunelords.com/youtubestatistics 2018

5. Wittich CM, Ficalora RD, Mason TG, Beckman TJ. Musculoskeletal injection. Mayo Clin Proc 2009; 84: 831-837.

6. Townsend RN, Clark R, Ramenofsky ML, Diamond DL. ATLS-based videotape trauma resuscitation review: education and outcome. J Trauma 1993; 34: 133-138.

7. Şaşmaz M, Akça A. Reliability of trauma management videos on YouTube and their compliance with ATLS ${ }^{\circledR}$ guideline. Eur J Trauma Emerg Surg 2018; 44: 753-757.

8. Cardone DA, Tallia AF. Diagnostic and therapeutic injection of the hip and knee. Am Fam Physician 2003; 67: 2147-2152.

9. Rastogi AK, Davis KW, Ross A, Rosas HG. Fundamentals of Joint Injection. AJR Am J Roentgenol 2016; 207: 484-494.

10. Weissenbach R. [Technic of injections into the knee]. Vie Med. 1963; 44: 113-114. French. PMID: 13999760

11. Elicabuk H, Yaylac1 S, Yilmaz A, Hatipoglu C, Kaya FG, Serinken M. The Reliability of Turkish ""'""""'"Basic Life Support""'"""'"" and """"'"" "Cardiac Massage"" " "'""" Videos Uploaded to Websites. Eurasian J Med 2016; 48: 15-19.

12. Ho M, Stothers L, Lazare D, Tsang B, Macnab A. Evaluation of educational content of YouTube videos relating to neurogenic 
bladder and intermittent catheterization. Can Urol Assoc J 2015; 9: 320-354.

13. Azer SA, AlGrain HA, AlKhelaif RA, AlEshaiwi SM. Evaluation of the Educational Value of YouTube Videos About Physical Examination of the Cardiovascular and Respiratory Systems. J Med Internet Res 2013; 15: e241.

14. Lee JS, Seo HS, Hong TH. YouTube as a potential training method for laparoscopic cholecystectomy. Ann Surg Treat Res 2015; 89: 92-97.

15. Rössler B, Lahner D, Schebesta K, Chiari A, Plöchl W. Medical information on the internet: quality assessment of lumbar puncture and neuroaxial block techniques on YouTube. Clin Neurol Neurosurg 2012; 114: 655-658.

16. Tourinho FSV, Medeiros KSd, Salvador PTCDO, Castro GLT, Santos VEP. Analysis of the YouTube videos on basic life support and cardiopulmonary resuscitation. Rev Col Bras Cir 2012; 39: 335-339.

17. Pitcher GS, Newton DH, Amendola MF. Common femoral artery access on YouTube: what practices are being shown and who is delivering the message? J Surg Educ 2017; 74: 455-458.
18. Nason GJ, Kelly P, Kelly ME, Burke MJ, Aslam A, Giri SK, et al. YouTube as an educational tool regarding male urethral catheterization. Scand J Urol 2015; 49: 189192.

19. Fischer J, Geurts J, Valderrabano V, Hügle T. Educational quality of YouTube videos on knee arthrocentesis. J Clin Rheumatol 2013; 19: 373-376.

20. Karim J, Marwan Y, Dawas A, Esmaeel A, Snell L. Learning knee arthrocentesis using YouTube videos. Clin Teach 2020; 17: 148152.

21. Kucukakkas O, Atar E, Berberoglu AH, Karaman CA, Korkmaz MB. YouTube as an information source for intra-articular knee injection. Annals of Medical Research 2019; 26: 918-923.

22. Berman JR, Ben-Artzi A, Fisher MC, Bass AR, Pillinger MH. A comparison of arthrocentesis teaching tools: cadavers, synthetic joint models, and the relative utility of different educational modalities in improving trainees' comfort with procedures. J Clin Rheumatol 2012; 18: 175-179.

23. Chenkin J, Lee S, Huynh T, Bandiera G. Procedures Can Be Learned on the Web: A Randomized Study of Ultrasound-guided Vascular Access Training. Acad Emerg Med 2008; 15: 949-954. 